\title{
Obstructive Jaundice in Polycystic Liver Disease Related to Coexisting Cholangiocarcinoma
}

\author{
Spiros G. Delis ${ }^{\mathrm{a}} \quad$ Andreas Bakoyiannis ${ }^{\mathrm{a}}$ \\ Charikleia Triantopoulou $^{\mathrm{b}}$ Kostantina Paraskeva $^{\mathrm{c}}$ \\ Kostas Athanassiou ${ }^{a} \quad$ Christos Dervenis $^{a}$ \\ aLiver Surgical Unit, ${ }^{b}$ Computed Tomography Department, and 'Department of \\ Gastroenterology, Agia Olga Hospital, Athens, Greece
}

\section{Key Words}

Polycystic liver disease $\cdot$ Cholangiocarcinoma $\cdot$ Jaundice $\cdot$ Bile duct compression

\begin{abstract}
Although jaundice rarely complicates polycystic liver disease (PLD), secondary benign or malignant causes cannot be excluded. In a 72-year-old female who presented with increased abdominal girth, dyspnea, weight loss and jaundice, ultrasound and computed tomography confirmed the diagnosis of PLD by demonstrating large liver cysts causing extrahepatic bile duct compression. Percutaneous cyst aspiration failed to relief jaundice due to distal bile duct cholangiocarcinoma, suspected by magnetic resonance cholangiopancreatography (MRCP) and confirmed by endoscopic retrograde cholangiopancreatography (ERCP). Coexistence of PLD with distal common bile duct cholangiocarcinoma has not been reported so far.
\end{abstract}

\section{Introduction}

Polycystic liver disease (PLD) is a genetic inherited entity associated with polycystic renal disease due to mutations in PKD1 or PKD2 genes [1]. A rarer PRKCSH gene mutation exists and is associated with isolated multiple liver cysts [2]. Symptoms are unspecific and are caused by increased liver volume and adjacent visceral compression. Surgical intervention is needed in cases presenting with massive hepatomegaly, or when complications such as cyst rupture, infection, hemorrhage or jaundice occur. Obstructive jaundice is a rare finding usually associated with secondary causes rather than external cyst compression of the bile ducts [3]. Although there are reports in the literature of cyst- 
associated jaundice $[4,5]$ our experience suggests that a thorough workup to exclude secondary causes such as gallstones and malignancy is mandatory.

\section{Case Report}

A 72-year-old Caucasian female presented with increased abdominal girth and jaundice. She complained of chronic dull pain, satiety, weight loss, dyspnea and physical disability. The patient had had clay-coloured stools and dark urine the last two weeks before admission.

Physical examination revealed a large non-tender abdominal mass and no signs of chronic liver disease such as splenomegaly or ascites.

Liver function tests were compatible with obstructive jaundice, with total bilirubin $8.07 \mathrm{mg} / \mathrm{dl}$ (normal range $<1 \mathrm{mg} / \mathrm{dl}$ ), with direct predominance $(7.51 \mathrm{mg} / \mathrm{dl}$ ) and elevation of alkaline phosphatase (337 U/l, normal range 50-136U/l) and $\gamma$-GT (404 U/l, normal range 15-85 U/l). Ca19-9 value was 224 $\mathrm{U} / \mathrm{ml}$ (normal range $0-37 \mathrm{U} / \mathrm{ml}$ ).

Ultrasound examination of the abdomen showed multiple liver cysts ranging from 1.2 to $13 \mathrm{~cm}$ in diameter and dilated intrahepatic biliary ducts. Computed tomography (CT) scan was performed using the standard protocol for hepatobiliary disease evaluation (slice thickness $3-5 \mathrm{~mm}$, arterial-portal venous and delayed phases after contrast administration). The multicystic nature of the disease was documented. The two dominant large liver cysts were located adjacent to the hilum.

Percutaneous drainage of the hilar cysts was performed to confirm that subsequent fenestration or enucleation will resolve jaundice. However the persistence of cholestasis and the mild dilatation of intrahepatic bile ducts in the post drainage CT evaluation (fig. 1) indicated the coexistence of a secondary cause. The patient underwent magnetic resonance imaging and magnetic resonance cholangiopancreatography (MRI-MRCP) that was compatible with the presence of possible neoplasmatic infiltration at the distal common bile duct, since duct compression was not marked enough to justify intrahepatic biliary dilatation and furthermore the distal segment of the common bile duct was not evident (fig. 2 ). Endoscopic retrograde cholangiopancreatography (ERCP) was performed (fig. 3). The abrupt termination of the middle segment of the common bile duct was indicative of neoplastic infiltration. Brushing followed and confirmed the diagnosis of cholangiocarcinoma.

Pylorus-preserving pancreato-duodenectomy with dominant liver cyst enucleation was performed. Histopathological examination revealed a biliary adenocarcinoma of the distal common bile duct. The postoperative period was uneventful and the patient was discharged on the 9th postoperative day. At six-month follow-up the patient is free of recurrence.

\section{Discussion}

PLD is inherited as an autosomal dominant trait presenting in adulthood. Autosomal dominant polycystic disease is genetically heterogeneous, with mutations in two distinct genes predisposing to the combination of renal and liver cysts (AD-PKD1 and AD-PKD2) [1]. Furthermore, Davila et al. [2] reported a second gene (SEC6) related to PLD. This gene encodes a component of the protein translocation machinery in the endoplasmatic reticulum.

The disease is more frequent in women, and hepatic cysts can increase dramatically in number and size through the child-bearing years of early and middle adult life. The cysts in PLD can also increase in size and number during pregnancy or simultaneously with the use of exogenous female steroid hormones [6]. In one study it was shown that the use of estrogen in women with autosomal dominant polycystic kidney disease may selectively increase the severity of hepatic cystic disease [7].

Symptoms are caused by increased liver volume and adjacent visceral compression. Usually patients suffer from chronic dull abdominal pain, satiety, weight loss, dyspnea, 
physical disability and descensus. Liver function tests are usually normal except for mild elevation in ALP or $\gamma$-GT. Jaundice may rarely exist due to compression of the common bile duct by the cysts [8]. In our experience, including this case, obstructive jaundice is usually associated with a co-existing underlying pathology such as gallstone disease or malignancy.

In PLD, liver failure or complications of advanced liver disease are rare. Even with marked hepatosplenomegaly and portal hypertension, liver function is well preserved. Thus jaundice is uncommon and requires further exploration to exclude benign or malignant disease. In our case the final diagnosis related to jaundice was a co-existing biliary adenocarcinoma. Other authors have reported co-existing choledocholithiasis and other complications of PLD causing obstructive jaundice [9].

In case of jaundice, standard pre and post contrast MRI sequences are necessary to establish the presence of an underlying malignancy that may be suggested by the biliary dilatation seen at MRCP. MRCP is also helpful to estimate the intraductal extent of tumor infiltration, facilitating surgical planning. ERCP can also help to confirm the cause of jaundice, presenting the further advantage of performing brushing or biopsy. The presence of the cholangiocarcinoma in the distal common bile duct, as was shown on imaging in our case, led the surgical team to perform a successful pylorus-preserving pancreatoduodenectomy with dominant liver cysts enucleation, which was considered the best therapeutic option for the patient.

Cholangiocarcinoma is the most common primary malignancy of the bile ducts and accounts for $15-50 \%$ of obstructing bile duct tumors [10]. Factors that predispose to the development of cholangiocarcinoma include ulcerative colitis, primary sclerosing cholangitis, choledochal cysts, Caroli disease, polyposis syndromes of the colon and Clonorchis sinensis infestation [10]. Cholangiocacinomas are generally classified as intrahepatic (peripheral) lesions, hilar lesions, and distal ductal tumors. CT appearance depends on their location, morphology and tumor pathology. If no tumor mass is seen, diagnosis of hilar cholangiocarcinoma can be made by the presence of high-grade intrahepatic bile duct dilatation and the absence of right and left hepatic duct union. If the neoplasm is located at a lower level, as in our case, at the point of obstuction the duct will terminate abruptly or there may be associated eccentric thickening of bile duct wall [1113]. The reason of the nonvisibility of the cholangiocarcinoma on CT images in our patient could be attributed to cyst superimposition obscuring the lesion and to the infiltrative type of the neoplasm, as was proven on histopathology, characterized by the absence of tumor mass.

MRCP is essential for the accurate estimation of obstruction level and the depiction of the characteristic morphologic features of stenosis or obstruction (abrupt termination of the bile duct as in our case) [14]. Because of their intrinsic high tissue contrast and multiplanar capability, MRI and MRCP are able to detect and preoperatively assess patients with cholangiocarcinoma, investigating all involved structures such as bile ducts, vessels and hepatic parenchyma. The main reason for surgical/imaging discrepancy is represented by the microscopic diffusion along the mucosa and in the perineural space. It should also be emphasized that the presence of multiple cystic liver lesions superimposed on the biliary ducts, displacing or distorting them, may obscure the presence of a cholangiocarcinoma. On the other hand, false-negative results are also encountered with ERCP washing or brushing in some patients due to the submucosal tumor growth.

Intrahepatic cholangiocarcinoma occurs rarely in patients with multiple liver cysts and tumor origin from cysts after in situ transformation of the epithelium has been shown in 
some cases [15-18]. The prognosis of cholangiocarcinoma associated with PLD is very poor and distant metastases are common. The possibility of cholangiocarcinoma should be considered in any patient with autosomal dominant polycystic kidney disease with significant abnormal liver function tests. After extensive review of the literature, it appears that coexistence of PLD with distal common bile duct cholangiocarcinoma as in our case has not been reported so far.

Less than 5\% of patients with PLD have acute medical complications. These consist of cyst hemorrhage, rupture, infection, uterine prolapse due to displacement, obstructive jaundice, portal hypertension, transudative and exudative ascites and Budd-Chiari syndrome $[19,20]$. Surgery is indicated in most cases, as medical treatment has failed to show any efficacy. Use of somatostatin to block the secretin-induced secretion by hepatic cysts failed to demonstrate any significant effect on hepatic cyst growth size [21]. Cyst aspiration with sclerosis, open or laparoscopic cyst fenestration, combined hepatic resection and fenestration or liver transplantation are possible treatment options. Treatment approaches should be individualized based on the type of PLD.

Aspiration combined with ethanol instillation to induce sclerosis of the cyst lining epithelium can be effective in patients with a few dominant cysts (type I PLD - few large cysts $>7 \mathrm{~cm}$ ). However, long-term recurrence presents in 50\% of cases [22]. Cyst fenestration can be performed in patients with more diffuse PLD (type II PLD - multiple medium cysts $5-7 \mathrm{~cm}$ in diameter). Morbidity after laparoscopic fenestration ranges between 33 and $45 \%$, with high recurrence rate. Patients with small cysts throughout the liver have a greater risk of persistence and recurrence of symptoms.

Combined hepatic resection and fenestration is more effective in reducing the hepatic mass and gastric compression. Recurrence rates vary significantly in the literature with high postoperative morbidity rates (38-100\%) [23]. This procedure offers an additional advantage in the case of massive hepatomegaly and severe symptoms of gastric compression. Resection addresses the problem of liver mass, but poses a significant risk of biliary ductal injury, vascular compromise, and liver insufficiency because cysts markedly distort the intrahepatic anatomy [24]. Postoperative morbidity is related to the presence of temporary ascites, pleural effusion and biliary leakage. In particular, ascites has been troublesome due to continued cyst secretion from residual fenestrated cysts, disruption of intrahepatic lymphatics and partial venous outflow obstruction. Candidates for combined resection/fenestration should have at least two adjacent liver segments not involved by cysts and normal liver function. These patients should be also managed by experienced hepatobiliary surgeons at institutions with advanced intensive care and interventional radiological and gastroenterology support.

Liver transplantation has been performed in rare cases when the above-mentioned interventions were not an option. In patients who harbour diffuse PLD, orthotopic liver transplantation is effective, but inherently assumes the risks of long-term immunosuppression and rejection. Orthotopic liver transplantation is indicated for patients with progressive PLD after resection/fenestration, patients with concurrent liver dysfunction and renal failure, and patients with diffuse PLD without segmental sparing [25]. 


\section{Conclusion}

Isolated PLD is a rare entity. Jaundice presents infrequently in this group of patients, unless a secondary cause exists. In such situations a thorough diagnostic workup is mandatory to exclude the coincidence of benign or malignant disease.

Fig. 1. Non-contrast CT image after percutaneous drainage of the two larger hepatic cysts. Mild intrahepatic biliary dilatation is evident (arrows).

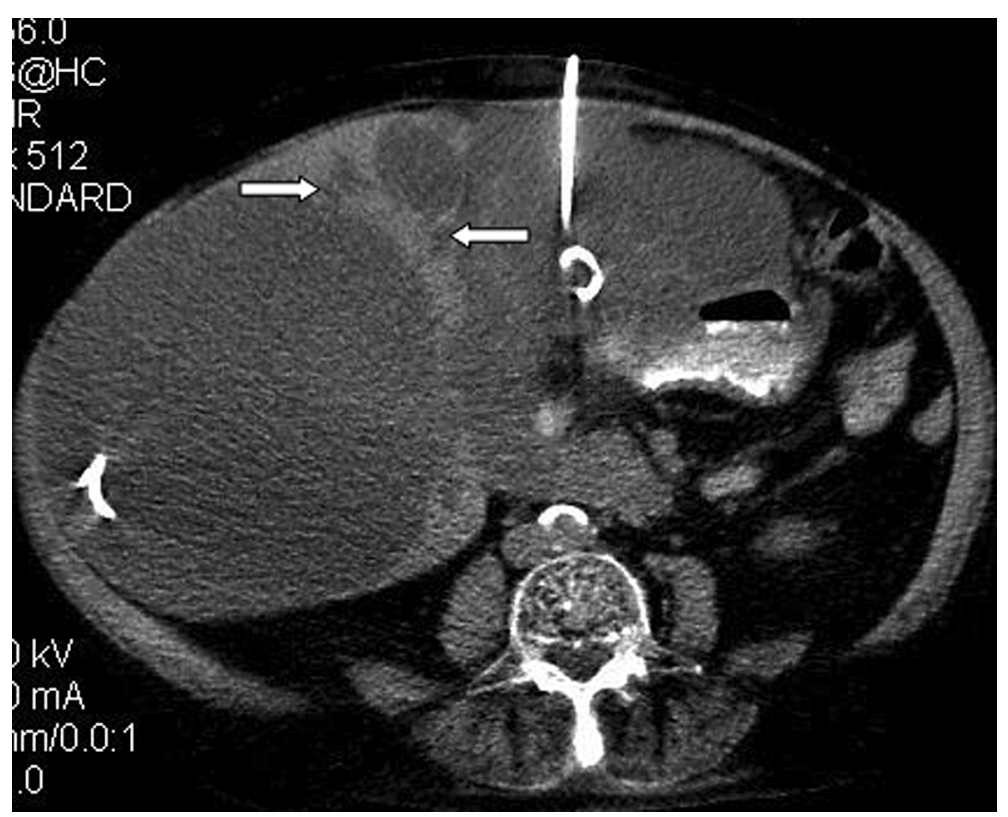


Fig. 2. MRCP image (T2-weighted sequence): multiple hepatic cysts are shown (arrows) while the common bile duct seems mildly compressed (arrowheads) between the two larger cysts. The distal segment of the common bile duct is not evident.

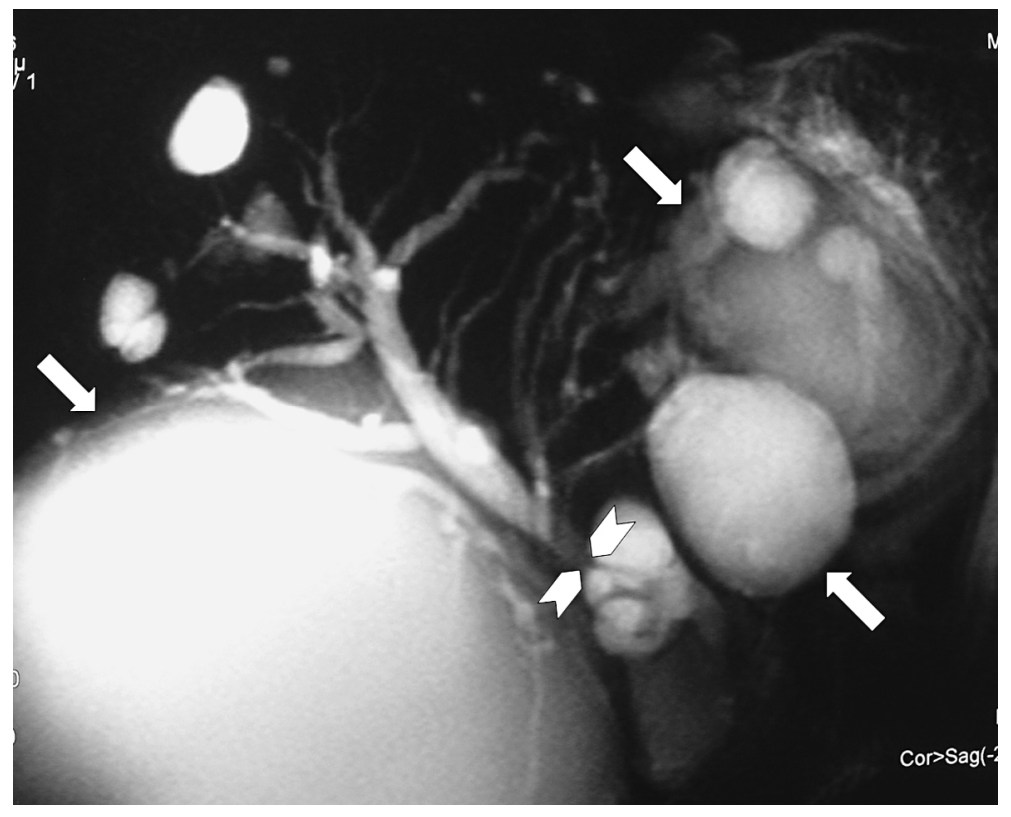

Fig. 3. ERCP image during the procedure of stent placement. Prominent intra- and extrahepatic bile duct dilatation with abrupt termination of the middle segment of the common bile duct (arrow) is shown consistent with neoplastic disease.

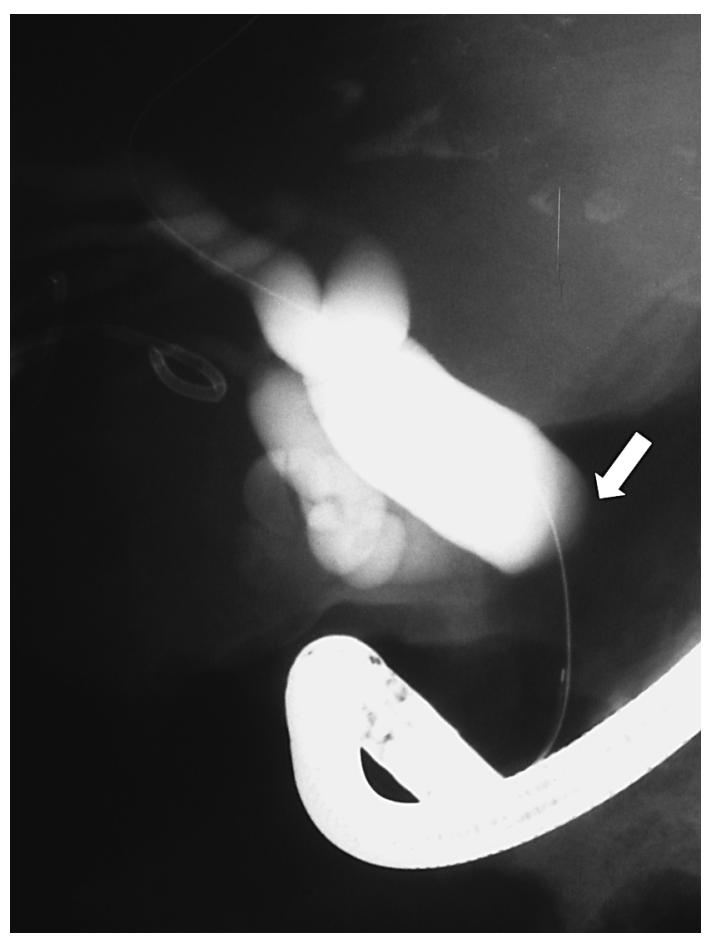




\section{References}

1 Everson GT, Taylor MR, Doctor RB: Polycystic disease of the liver. Hepatology 2004;40:774-782.

2 Davila S, Furu L, Gharavi AG, et al: Mutations in SEC63 cause autosomal dominant polycystic liver disease. Nat Genet 2004;36:575-577.

-3 Levine E, Cook LT, Grantham JJ: Liver cysts in autosomal-dominant polycystic kidney disease: Clinical and computed tomographic study. AJR 1985;145:229233.

-4 Ergun $\mathrm{H}$, Wolf BH, Hissong SL: Obstructive jaundice caused by polycystic liver disease. Radiology 1980;136:435-436.

5 Wittig JH, Burns R, Longmire WP: Jaundice associated with polycystic liver disease. Am J Surg 1978;136:383-386.

-6 Everson GT, Taylor MR: Management of polycystic liver disease. Curr Gastroenterol Rep 2005;7:19-25.

7 Sherstha R, McKinley C, Russ P, et al: Postmenopausal estrogen therapy selectively stimulates hepatic enlargement in women with autosomal dominant polycystic kidney disease. Hepatology 1997;26:1282-1286.

8 Rosenfeld L, Bonny C, Kallita M, Heng AE, Deteix P, Bommelaer G, Abergel A: Polycystic liver disease and its main complications. Gastroenterol Clin Biol 2002;26:1097-1106.

>9 Bistritz L, Tomboli C, Bigam D, Bain VG: Polycystic liver disease: experience at a teaching hospital. Am J Gastroenterol 2005;100:1-7.

10 D'Angelica JM, Blumgart LH: Intrahepatic and extrahepatic biliary cancer; in Blumgart LH (ed): Surgery of the Liver Biliary Tract and Pancreas. Philadelphia, Saunders, 2007, pp 782-822.

11 Lee WJ, Lim HK, Jank KM, et al: Radiologic spectrum of cholangiocarcinoma: emphasis on unusual manifestations and differential diagnoses. Radiographics 2001;21:S97-S116.

12 Zech C, Schoenberg SO, Reiser M, et al: Cross-sectional imaging of biliary tumors: current clinical status and future developments. Eur Radiol 2004;14:1174-1187.

13 Han JK, Choi BI, Kim AH, et al: Cholangiocarcinoma: pictorial essay of CT and cholangiographic findings. Radiographics 2002;22:173-187.

14 Dranssart M, Cognet F, Mousson C, Cercueil JP, Rifle G, Krause D: MR cholangiography in the evaluation of hepatic and biliary abnormalities in autosomal dominant polycystic kidney disease: study of 93 patients. J Comput Assist Tomogr 2002;26:237-242.

15 Grunfeld JP, Albouze G, Jungers P, et al: Liver changes and complications in adult polycystic kidney disease. Adv Nephrol 1985;14:1-20.

16 Son JH, Kwon SY, Song SW, et al: A case of intrahepatic cholangiocarcinoma in polycystic liver disease. Korean J Hepatol 1999;5:156-161.

17 Landais P, Grunfeld JP, Droz D, et al: Cholangiocellular carcinoma in polycystic kidney and liver disease. Arch Intern Med 1984;144:2274-2276.

18 Sasaki M, Katayanagi K, Watanabe K, Takasawa K, Nakanuma Y: Intrahepatic cholangiocarcinoma arising in autosomal dominant polycystic kidney disease. Virchows Arch 2002;441:98-100.

19 Arnold HL, Harrison SA: New advances in evaluation and management of patients with polycystic liver disease. Am J Gastroenterol 2005;100:2569-2582.

-20 Brancatelli G, Federle MP, Vilgrain V, Vullieme MP, Marin D, Lagala R: Fibropolycystic liver disease: CT and MR imaging findings. Radiographics 2005;5:659-670.

21 Chauveau D, Martinez F, Grunfeld JP: Evaluation of octreotide in massive polycystic liver disease. 12th international Congress of Nephrology Conference Program, 1993, p 487A.

22 Tikkakoski T, Makela JT, Leinonen S, et al: Treatment of symptomatic congenital hepatic cysts with single-session percutaneous drainage and ethanol sclerosis: Technique and outcome. J Vasc Interv Radiol 1996;7:35-39.

23 Iwatsuki S, Starzl TE: Personal experience with 411 hepatic resections. Ann Surg 1988;208:421-434. 
24 Que F, Nagorney DM, Gross JB Jr, Torres VE: Liver resection and cyst fenestration in the treatment of severe polycystic liver disease. Gastroenterology 1995;108:487-494.

25 Gustafsson BI, Friman S, Mjornstedt L, Olausson M, Backman L: Liver transplantation for polycystic liver disease - indications and outcome. Transplant Proc 2003;35:813-814. 